\title{
On the tidally induced gravitational collapse of a particle cluster
}

\author{
Kashif Alvi* $\quad$ Yuk Tung Liu*
}

\begin{abstract}
An important issue in the dynamics of neutron star binaries is whether tidal interaction can cause the individual stars to collapse into black holes during inspiral. To understand this issue better, we study the dynamics of a cluster of collisionless particles orbiting a non-rotating black hole, which is part of a widely separated circular binary. The companion body's electric- and magnetic-type tidal fields distort the black hole and perturb the cluster, eventually causing the cluster to collapse into the hole as the companion spirals in under the influence of gravitational radiation reaction. We find that magnetic-type tidal forces do not significantly influence the evolution of the cluster as a whole. However, individual orbits can be strongly affected by these forces. For example, some orbits are destabilized due to the addition of magnetic-type tidal forces. We find that the most stable orbits are close to the companion's orbital plane and retrograde with respect to the companion's orbit.
\end{abstract}

\section{Introduction}

Gravitational wave detectors will soon begin operation and may see signals from sources such as binary neutron stars. An important issue in the dynamics of these binaries is whether tidal interaction between the neutron stars induces each one to collapse to form a black hole during the late stages of inspiral ([1] and references therein). Recent numerical simulations have shown that the stars either have a very slight tendency towards individual collapse or are completely stable to such collapse (see e.g. [1, 2, 3]). Given the complexity of relativistic hydrodynamic simulations, it is useful to study simpler models in order to understand the physical underpinnings of tidally induced collapse (or stability against collapse) in neutron star binaries.

One such model was considered by Shapiro and collaborators [4, 57. It consists of a cluster of collisionless particles orbiting a non-rotating black hole, which is part of a widely separated circular binary. They found that as the hole's companion body spirals in slowly, its tidal field induces inward collapse (into the hole) of the cluster. However, they restricted attention to the effect of the companion body's electric-type tidal field. In the real physical situation of binary neutron stars, magnetic-type tidal fields may play an important role in the stability of the stars [6]. The purpose of this paper is to investigate the effect of the companion body's magnetic-type tidal field on the evolution of the particle cluster.

\section{Framework}

Consider a binary system composed of a non-rotating black hole of mass $m_{1}$ and a companion body of mass $m_{2}$. Let $m=m_{1}+m_{2}$ and $\mu=m_{1} m_{2} / m$. Denote the binary's orbital separation by

\footnotetext{
${ }^{*}$ Theoretical Astrophysics 130-33, California Institute of Technology, Pasadena, California 91125
} 
$b$. Define the following Newtonian quantities: the relative velocity $v=(m / b)^{1 / 2}$, and the orbital angular velocity

$$
\omega_{N}=\left(m / b^{3}\right)^{1 / 2}
$$

By assumption, $m \ll b$, so $v \ll 1$.

Assume the binary is in a circular orbit that decays due to emission of Newtonian quadrupole gravitational radiation. This means the separation $b$ evolves as [7, 8]

$$
b(t)=b_{0}\left(1-t / \tau_{0}\right)^{1 / 4}
$$

where $\tau_{0}=(5 / 256) b_{0}^{4}\left(\mu m^{2}\right)^{-1}$.

Since the binary is widely separated, the region near the black hole can be described by a tidally perturbed Schwarzschild metric. Cover this region by Schwarzschild coordinates $(t, r, \theta, \phi)$. Additional coordinate systems that will be useful are isotropic coordinates $(t, x, y, z)$ and spherical isotropic coordinates $(t, R, \theta, \phi)$, which are related to Schwarzschild coordinates via

$$
r=R\left(1+m_{1} / 2 R\right)^{2}, \quad x=R \sin \theta \cos \phi, \quad y=R \sin \theta \sin \phi, \quad z=R \cos \theta .
$$

Place a set of test particles at random positions on the sphere $r=r_{c} \ll b$ and let their initial velocities be in random directions tangent to the sphere. In the test particle approximation, each particle moves along a geodesic of the tidally perturbed Schwarzschild metric. Set the magnitude of each particle's initial velocity to yield a circular orbit when $m_{2}=0$. Determine the cluster's time evolution by integrating the geodesic equation and assuming the particles do not collide.

\section{Estimate of magnetic-type tidal force}

Given a $3+1$ split of spacetime or a local reference frame, one can separate the ten independent components of the Weyl tensor into an electric- and a magnetic-type tidal tensor, each having five independent components (see e.g. Secs. V.A.2-3 in [9]). We are interested in the companion body's tidal fields as seen by an inertial observer in the black hole's local asymptotic rest frame [10]. This observer sees an electric-type tidal field whose typical components have magnitude $\mathcal{E} \sim m_{2} / b^{3}$, where " " means "is of the order of". If the companion body was stationary with respect to the black hole, the observer would not see a magnetic-type tidal field. The motion of the companion at velocity $v$ with respect to the black hole induces, via a Lorentz boost with velocity $v$, a magnetictype tidal field whose typical components have magnitude $\mathcal{B} \sim v \mathcal{E}$ as seen by the inertial observer [11.

The magnitude of the magnetic-type tidal force experienced by a particle moving in the local asymptotic rest frame is of order $m_{p} v_{p} r \mathcal{B} \sim m_{p} v_{p} v r \mathcal{E}$ [12], where $m_{p}$ is the particle's mass, $v_{p}$ is the particle's velocity as measured by the inertial observer, and $r$ is the particle's orbital radius. This is smaller by a factor of $v_{p} v$ than the electric-type tidal force $m_{p} r \mathcal{E}$.

Now consider a particle cluster at $r=r_{c}$. The representative particle velocity of this cluster is $v_{p}=\left(m_{1} / r_{c}\right)^{1 / 2}$. Shapiro and collaborators set $r_{c}=6.9 m_{1}$ and $m_{1}=m_{2}$, and observe cluster collapse when the companion body reaches $b \approx 15 m[5]$. At that point, $v_{p} v=\left(m_{1} m / r_{c} b\right)^{1 / 2} \approx 0.1$, so the magnetic-type tidal forces on the particles are a significant fraction of the electric ones. Furthermore, the two types of tidal forces typically point in different directions. Therefore, the magnetic-type tidal field could have an important effect on the dynamics of the cluster. In the next sections, we investigate this issue. 


\section{Tidal perturbation}

The leading order electric- and magnetic-type tidal deformations of a non-rotating black hole in a widely separated circular binary have been calculated in [11] using black hole perturbation theory. The tidally perturbed metric near the black hole is given in Schwarzschild coordinates $(t, r, \theta, \phi)$ by (see Sec. III in [1])

$$
\begin{aligned}
d s^{2}= & -\left(1-\frac{2 m_{1}}{r}\right) d t^{2}+\left(1-\frac{2 m_{1}}{r}\right)^{-1} d r^{2}+r^{2}\left(d \theta^{2}+\sin ^{2} \theta d \phi^{2}\right) \\
& -\frac{4 m_{2}}{b^{3}} \sqrt{\frac{m}{b}}\left(1-\frac{2 m_{1}}{r}\right) r^{3} d t[\cos \theta \sin (\phi-\omega t) d \theta+\sin \theta \cos (2 \theta) \cos (\phi-\omega t) d \phi] \\
& +\frac{m_{2} r^{2}}{b^{3}}\left[3 \sin ^{2} \theta \cos ^{2}(\phi-\omega t)-1\right]\left[\left(1-\frac{2 m_{1}}{r}\right)^{2} d t^{2}+d r^{2}\right. \\
& \left.+\left(r^{2}-2 m_{1}^{2}\right)\left(d \theta^{2}+\sin ^{2} \theta d \phi^{2}\right)\right],
\end{aligned}
$$

where

$$
\omega=\omega_{N}\left(1-\frac{\mu}{b}\right)
$$

The metric perturbation has been written in Regge-Wheeler gauge 13] and the orbital plane of the companion has been taken, without loss of generality, to be the black hole's equatorial plane. The rotation rate $\omega$ of the companion body's tidal field, as measured by an inertial observer in the black hole's local asymptotic rest frame, differs from the Newtonian orbital angular velocity $\omega_{N}$ because of precession effects [11.

The magnetic-type tidal perturbation on the black hole is embodied in the metric components $g_{t \theta}$ and $g_{t \phi}$ in (4). Setting $g_{t \theta}=0=g_{t \phi}$ yields the metric utilized in [5]; this metric contains only the electric-type tidal perturbation. In what follows, we sometimes switch off the magnetic-type tidal perturbation by setting $g_{t \theta}=0=g_{t \phi}$, in order to isolate magnetic-type tidal effects and to compare with the results in [5].

If we ignore radiation reaction, the binary's orbit remains circular and $\omega$ is constant. In this situation, the vector field $\vec{K}=\frac{\partial}{\partial t}+\omega \frac{\partial}{\partial \phi}$ is a Killing field of the metric (ब14), and $\vec{K} \cdot \vec{u}$ is constant along each particle's geodesic worldline, where $\vec{u}$ is the particle's four-velocity. This constancy serves as an important check on our numerics. Indeed, we first turned off radiation reaction and verified $\vec{K} \cdot \vec{u}$ was constant to sufficient accuracy along particle orbits.

In our actual simulations, we include the effects of radiation reaction on the binary by substituting $b(t)$ from Eq. (2) into (1) and (5). We also replace $\omega t$ in (4) by $\psi(t)=\int_{0}^{t} \omega\left(t^{\prime}\right) d t^{\prime}$, where $\omega(t)$ is given by (5), (11), and (2). Furthermore, we transform (位) to isotropic coordinates $(t, x, y, z)$ in order to avoid difficulties with spherical coordinates. This yields

$$
\begin{aligned}
& g_{t t}=-\left(\frac{1-m_{1} / 2 R}{1+m_{1} / 2 R}\right)^{2}+\frac{m_{2}}{b^{3}}\left(1-\frac{m_{1}}{2 R}\right)^{4}\left[3(x \cos \psi+y \sin \psi)^{2}-R^{2}\right], \\
& g_{t x}=\frac{2 m_{2}}{b^{3}} \sqrt{\frac{m}{b}}\left(1-\frac{m_{1}}{2 R}\right)^{2}\left(1+\frac{m_{1}}{2 R}\right)^{4}\left[\left(z^{2}-y^{2}\right) \sin \psi-x y \cos \psi\right], \\
& g_{t y}=\frac{2 m_{2}}{b^{3}} \sqrt{\frac{m}{b}}\left(1-\frac{m_{1}}{2 R}\right)^{2}\left(1+\frac{m_{1}}{2 R}\right)^{4}\left[\left(x^{2}-z^{2}\right) \cos \psi+x y \sin \psi\right], \\
& g_{t z}=\frac{2 m_{2}}{b^{3}} \sqrt{\frac{m}{b}}\left(1-\frac{m_{1}}{2 R}\right)^{2}\left(1+\frac{m_{1}}{2 R}\right)^{4}(y \cos \psi-x \sin \psi) z,
\end{aligned}
$$




$$
\begin{aligned}
g_{i j}= & \left(1+\frac{m_{1}}{2 R}\right)^{4}\left(\delta_{i j}+\frac{m_{2}}{b^{3}}\left[3(x \cos \psi+y \sin \psi)^{2}-R^{2}\right]\right. \\
& \left.\times\left\{\left[\left(1+\frac{m_{1}}{2 R}\right)^{4}-\frac{2 m_{1}^{2}}{R^{2}}\right] \delta_{i j}-\frac{2 m_{1}}{R}\left(1+\frac{m_{1}^{2}}{4 R^{2}}\right) \frac{x^{i} x^{j}}{R^{2}}\right\}\right),
\end{aligned}
$$

where $R=\left(x^{2}+y^{2}+z^{2}\right)^{1 / 2}$.

The particles in the cluster follow geodesics of this metric. We numerically integrate the geodesic equation

$$
\ddot{x}^{\alpha}+\Gamma_{\sigma \lambda}^{\alpha} \dot{x}^{\sigma} \dot{x}^{\lambda}=0
$$

to obtain the cluster's time evolution. Here, an overdot represents a derivative with respect to proper time.

\section{$5 \quad$ Numerical implementation and results}

We integrate Eq. (7) using a fourth order Runge-Kutta method with adaptive stepsize control 14. At $t=0$, we place the companion at $b=60 m_{1}$ and put 1000 particles at Schwarzschild radius $r_{c}=6.9 m_{1}$. We also set $m_{1}=m_{2}$. In computing the Christoffel symbols $\Gamma_{\sigma \lambda}^{\alpha}$, we ignore time derivatives of $b(t)$. This is consistent with the treatment in [5] and is justified by the wideseparation/slow-inspiral assumption.

We followed the cluster's evolution until $b=15 m_{1}\left(t=126068 m_{1}\right)$. To check the accuracy of our code, we turned off radiation reaction (i.e. set $b$ to be a constant) and verified that $\vec{u} \cdot \vec{u}$ and $\vec{K} \cdot \vec{u}$ were conserved along particle trajectories to a fractional accuracy of $10^{-7}$ during the entire evolution (i.e. from $t=0$ to $t=126068 m_{1}$ ).

The results of the simulation are presented in Fig. 1. The cluster's average radius $\langle r\rangle$ is plotted against the binary's separation $b$ for two cases: first, the magnetic-type tidal field and its distortion of the black hole are ignored, i.e. the metric components $g_{t i}$ in (6) are set to zero (dashed line); and second, both electric- and magnetic-type tidal fields are included, i.e. the full metric (6) is used (solid line). The dashed line reproduces the corresponding result in [5]. The dynamics of the cluster as a whole is quite similar in the two cases, even though individual orbits can be affected significantly by the magnetic-type tidal perturbation (see below). A large number of particles plunge into the black hole at $b \approx 30 m_{1}$, causing a sudden decrease of $\langle r\rangle$ (as seen in the figure).

It was observed in [5] that particles initially orbiting close to the equatorial plane (which is also the companion's orbital plane) are more stable against collapse into the black hole than those on initially polar orbits. Here we verify this observation in the presence of magnetic-type tidal forces. For each particle that plunges into the black hole by the end of our simulation, let $t_{\text {plunge }}$ denote the time at which the particle's orbital radius is $0.51 m_{1}\left(2.000196 m_{1}\right)$ in isotropic (Schwarzschild) coordinates, and let $b_{\text {plunge }}=b\left(t_{\text {plunge }}\right)$. For each particle, let $\iota$ denote its initial orbital inclination angle with respect to the equatorial plane. This quantity is defined by

$$
\cos \iota=\left.R^{-1}(x \dot{y}-y \dot{x})\left(\dot{x}^{2}+\dot{y}^{2}+\dot{z}^{2}\right)^{-1 / 2}\right|_{t=0} .
$$

On an unperturbed Schwarzschild background, this definition reduces to $\cos \iota=L_{z} / L$, where $L_{z}$ is the $z$-component of the particle's angular momentum and $L$ is its total angular momentum (both quantities are conserved along particle trajectories).

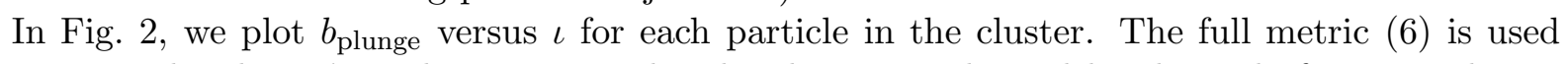
to compute the cluster's evolution. Particles that have not plunged by the end of our simulation are represented by squares and are placed on the $x$-axis. Particles that plunge in the presence of 


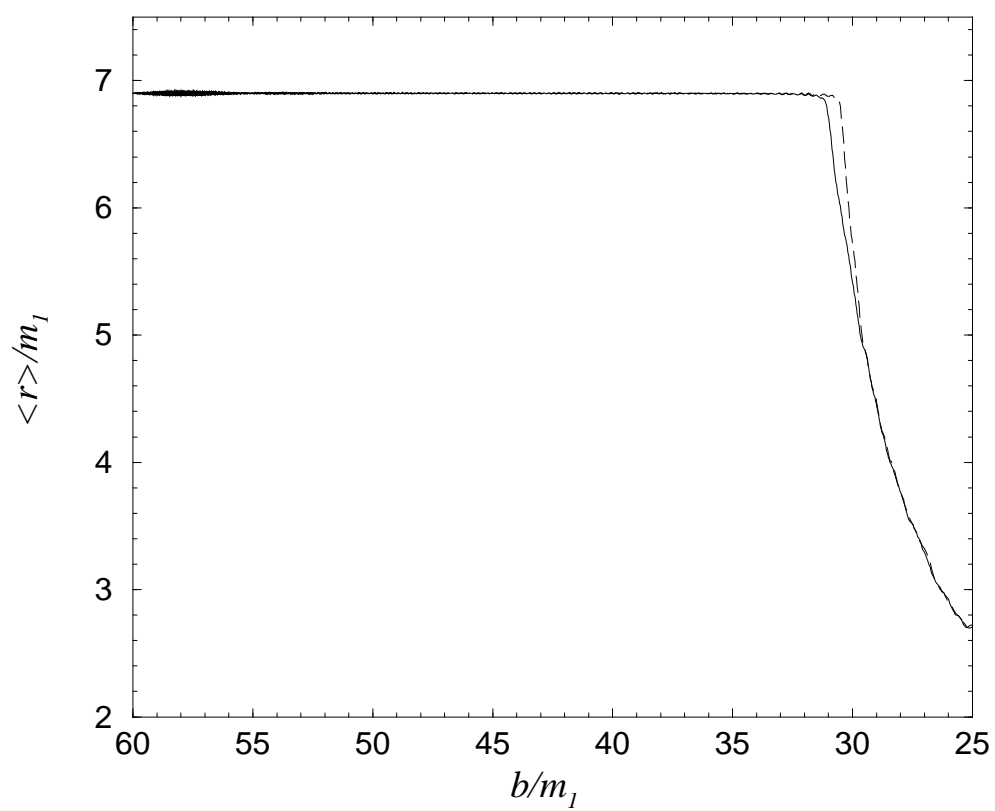

Figure 1: Cluster's average radius $\langle r\rangle$ as a function of binary separation $b$. The solid line represents evolution with both electric- and magnetic-type tidal perturbations present, and the dashed line with only electric-type present.

the magnetic-type tidal perturbation but do not plunge in its absence are represented by triangles. There are no particles that plunge in the absence of the magnetic-type tidal perturbation but do not plunge in its presence. The rest of the particles are represented by small circles.

A general trend in Fig. 2 is that the smaller the value of $\sin \iota$, the more stable the orbit. Orbits with $60^{\circ} \lesssim \iota \lesssim 120^{\circ}$ (nearly polar) are the least stable, while orbits with $\iota \gtrsim 165^{\circ}$ (retrograde with respect to the companion's orbit and nearly equatorial) are the most stable. Note that magnetictype tidal forces induce collapse in some particles that were stable in the absence of these forces.

In order to study the effect of magnetic-type tidal forces on individual orbits, we computed the time difference $\Delta t=t_{\text {plunge }}^{e m}-t_{\text {plunge }}^{e}$, where $t_{\text {plunge }}^{e m}\left(t_{\text {plunge }}^{e}\right)$ is the plunge time with (without) the magnetic-type tidal perturbation on the black hole, for particles that plunge in both cases by the end of our simulation. This time difference is plotted as a function of the inclination angle $\iota$ in Fig. 3. It is clear from the figure that the magnetic-type tidal force can have a significant influence on individual orbits.

For $50^{\circ} \lesssim \iota \lesssim 140^{\circ}$, the values of $\Delta t$ are roughly integral multiples of

$$
T_{r}=2 \pi r\left(\frac{r}{m_{1}}\right)^{1 / 2}\left(1-\frac{6 m_{1}}{r}\right)^{-1 / 2}
$$

which is the radial oscillation period of a nearly circular orbit outside $r=6 m_{1}$ (see also Fig. (4). For $\iota \lesssim 30^{\circ}$, the plunge occurs at smaller values of $b$ (see Fig. 2) and hence the particles experience stronger tidal forces. As a result, the amplitudes of the particles' radial oscillations are large and the formula (9) for nearly circular orbits is no longer valid. In Fig. 3, there are no points with $\iota \gtrsim 150^{\circ}$ because no particles with $\iota \gtrsim 150^{\circ}$ plunge in the absence of the magnetic-type tidal perturbation (see Fig. 2).

The last few radial oscillation periods before plunge for four selected particles are shown in Fig. 1 . The vertical axis is the Schwarzschild radius of the particle. The solid line represents 


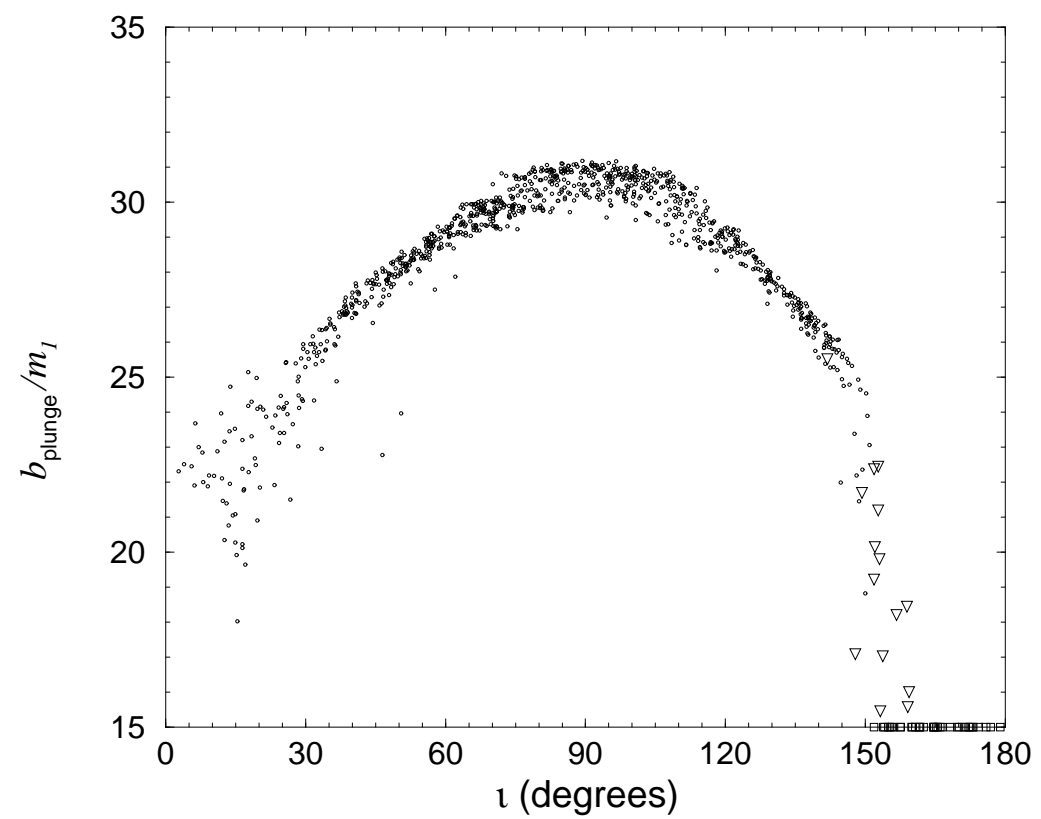

Figure 2: Binary separation at plunge, $b_{\text {plunge }}$, versus initial orbital inclination angle $\iota$ for each particle. Both electric- and magnetic-type tidal perturbations are included. Squares are particles that do not plunge; they are placed on the $x$-axis. Triangles are particles that are stable in the absence of the magnetic-type tidal perturbation.

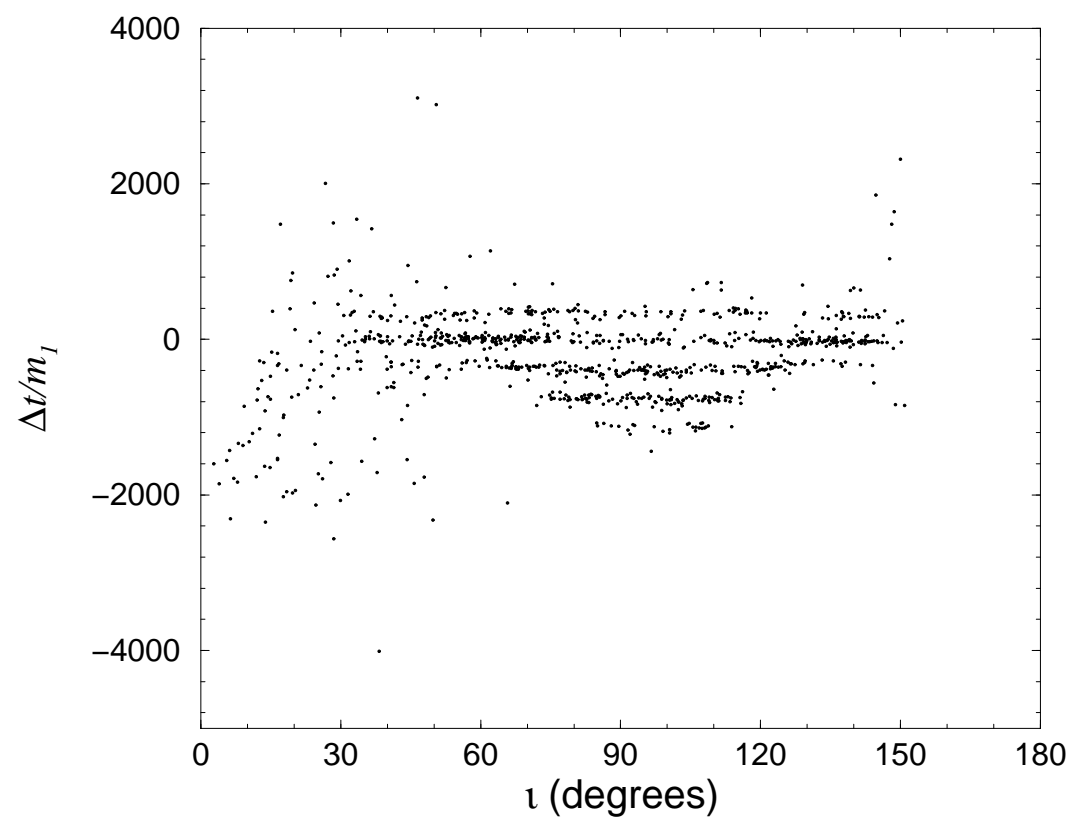

Figure 3: Difference $\Delta t$ in plunge times with and without magnetic-type tidal forces, plotted against initial orbital inclination angle $\iota$. Positive values of $\Delta t$ correspond to a delayed plunge due to magnetic-type tidal forces. 

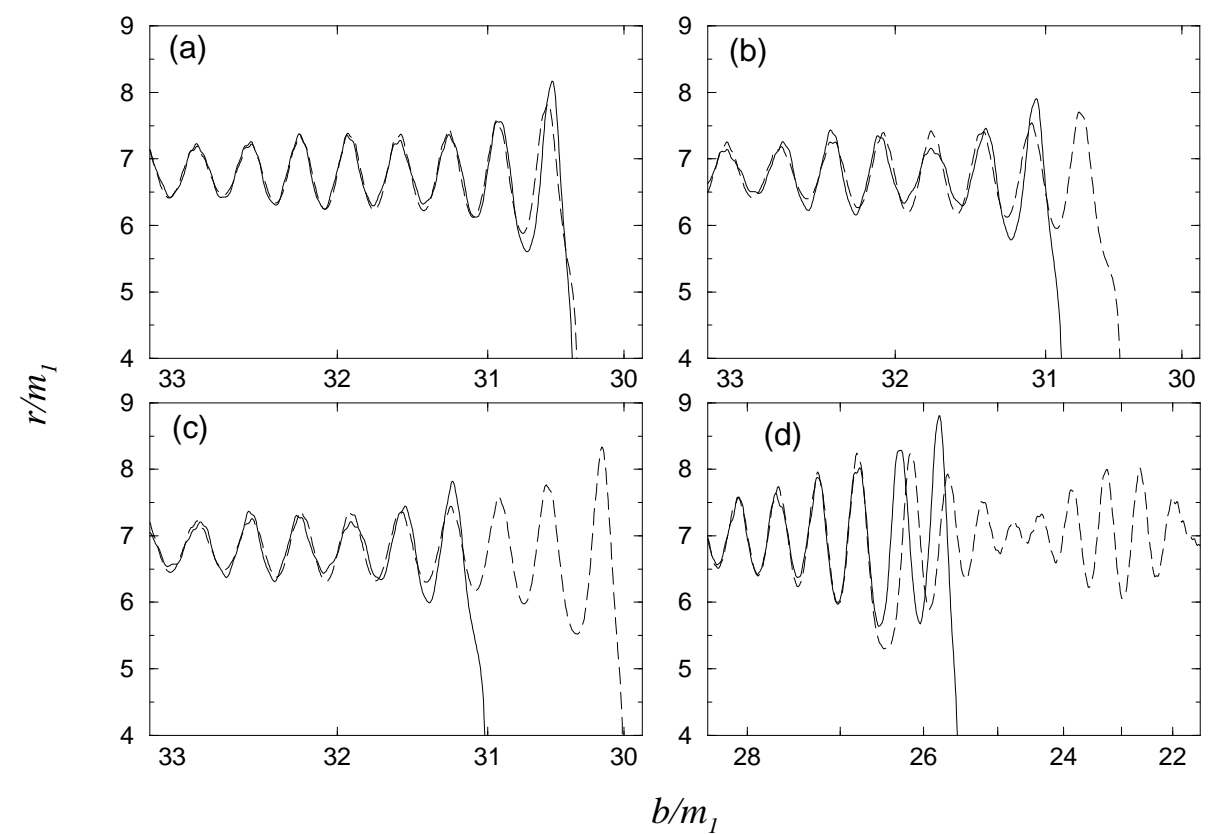

Figure 4: Radial oscillations before plunge for four selected particles: (a) $\iota=81.4^{\circ}$, (b) $\iota=89.9^{\circ}$, (c) $\iota=89.6^{\circ}$, and (d) $\iota=142^{\circ}$. The vertical axis is the Schwarzschild radius of the particle and the solid (dashed) lines represent motion with (without) magnetic-type tidal forces.

motion in the presence of both electric- and magnetic-type tidal perturbations, and the dashed line in the presence of only electric. Cases (a), (b), and (c) correspond to particles with $\Delta t / T_{r} \approx 0,-1$, and -3 respectively. Case $(\mathrm{d})$ is an example of a particle that is stable (unstable) in the absence (presence) of the magnetic-type tidal perturbation.

We varied the initial cluster radius $r_{c}$ to investigate how this parameter affects the system's evolution. Our results are displayed in Fig. 5. The cluster's average radius $\langle r\rangle$ is plotted against binary separation $b$ for $r_{c}=6.5,6.7,7.0$, and 7.2. We put 300 particles in the cluster for each of these simulations. Once again, solid (dashed) lines represent motion with (without) the magnetictype tidal perturbation. Cluster collapse occurs in all cases and is qualitatively similar with and without magnetic-type tidal forces.

Our simulations indicate that magnetic-type tidal forces do not significantly influence the dynamics of the particle cluster as a whole, though individual orbits can be strongly affected.

\section{Acknowledgments}

We are grateful to Kip Thorne and Lior Burko for useful comments and conversations. This research was supported in part by NSF grants PHY-0099568 and PHY-9796079, and NASA grants NAG5-10707 and NAG5-4093.

\section{References}

[1] G.J. Mathews and J.R. Wilson, Phys. Rev. D 61, 127304 (2000); gr-qc/9911047. 


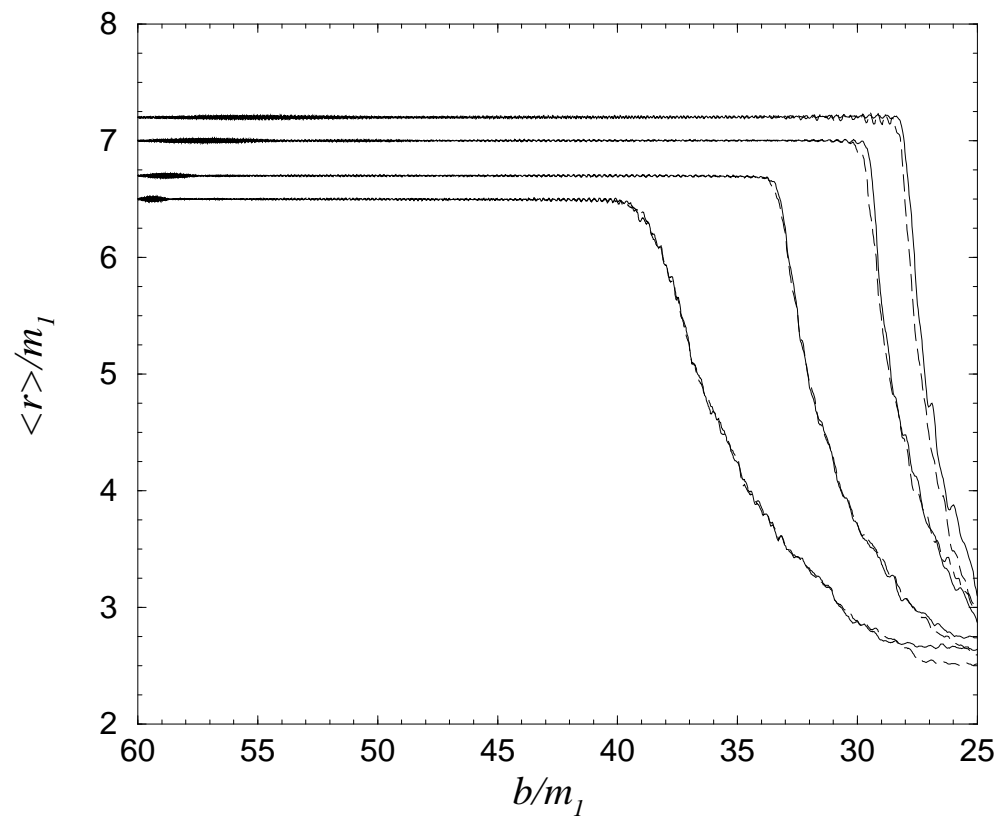

Figure 5: Cluster's average radius $\langle r\rangle$ as a function of binary separation $b$ for $r_{c}=6.5,6.7,7.0$, and 7.2 (with 300 particles in the cluster). The solid line represents evolution with both electricand magnetic-type tidal perturbations present, and the dashed line with only electric-type present.

[2] S. Bonazzola, E. Gourgoulhon, and J.-A. Marck, Phys. Rev. Lett. 82, 892 (1999); grqc/9810072.

[3] K. Uryu and Y. Eriguchi, Phys. Rev. D 61, 124023 (2000); gr-qc/9908059.

[4] S.L. Shapiro, Phys. Rev. D 57, 908 (1998); gr-qc/9710094.

[5] M.D. Duez et al., Phys. Rev. D 60, 104024 (1999); gr-qc/9904011.

[6] M. Favata and K.S. Thorne (in preparation).

[7] P.C. Peters, Phys. Rev. 136, B1224 (1964).

[8] C.W. Misner, K.S. Thorne, and J.A. Wheeler, Gravitation (Freeman, San Francisco, 1973).

[9] Black Holes: The Membrane Paradigm, edited by K.S. Thorne, R.H. Price, and D.A. Macdonald (Yale University Press, New Haven, 1986).

[10] K.S. Thorne and J.B. Hartle, Phys. Rev. D 31, 1815 (1985).

[11] K. Alvi, Phys. Rev. D 61, 124013 (2000); gr-qc/9912113.

[12] W.-T. Ni and M. Zimmermann, Phys. Rev. D 17, 1473 (1978).

[13] T. Regge and J.A. Wheeler, Phys. Rev. 108, 1063 (1957).

[14] W.H. Press, S.A. Teukolsky, W.T. Vetterling, and B.P. Flannery, Numerical Recipes in Fortran 77 (Cambridge University Press, New York, 1992). 